Article

\title{
Efficacy of Olanzapine for High and Moderate Emetogenic Chemotherapy in Children
}

\author{
So Rae Lee ${ }^{1,+}$, Su Min Kim ${ }^{1,+}$, Min Young $\mathrm{Oh}^{1,+}$ and Jae Min Lee ${ }^{2, *(1)}$ \\ 1 Department of Medicine, College of Medicine, Yeungnam University, Daegu 42415, Korea; \\ dlthfo@naver.com (S.R.L.); gogo_sumin@naver.com (S.M.K.); polarbear_yn@naver.com (M.Y.O.) \\ 2 Department of Pediatrics, College of Medicine, Yeungnam University, Daegu 42415, Korea \\ * Correspondence: mopic@hanmail.net; Tel.: +82-53-620-3536 \\ + So Rae Lee, Su Min Kim, and Min Young Oh contributed equally to this study.
}

Received: 21 August 2020; Accepted: 15 September 2020; Published: 16 September 2020

\begin{abstract}
This study was conducted to investigate the safety and efficacy of olanzapine for high and moderate emetogenic chemotherapy in children and young adults. We retrospectively reviewed the records of pediatric patients $(n=13)$ with cancer who had been administered olanzapine as an anti-emetic drug (AED) during a high and moderate emetogenic chemotherapy block from January 2018 to March 2020. Patients were administered other prophylactic AEDs according to practice guidelines. The mean age of the patients was $14.1 \pm 5.5$ years. The total number of chemotherapy cycles was 41 . Twenty-one (51.2\%) chemotherapy blocks were high emetogenic chemotherapy and $20(48.8 \%)$ blocks were moderate emetogenic chemotherapy. Olanzapine was used for prophylaxis in $20(48.8 \%)$ blocks of chemotherapy and rescue in $21(51.2 \%)$. Of the 41 cycles, a complete response to olanzapine was achieved in $31(75.6 \%)$, partial response in $6(14.6 \%)$, and no response in $4(9.8 \%)$. The mean dose was $0.07 \pm 0.04 \mathrm{mg} / \mathrm{kg} /$ dose and $2.50 \pm 1.37 \mathrm{mg} / \mathrm{m}^{2} /$ dose. Adverse effects included somnolence, hyperglycemia, fatigue, and disturbed sleep. Our findings indicate that olanzapine was effective and safe for treating chemotherapy-induced nausea and vomiting in children. A prospective controlled study is needed to confirm these findings.
\end{abstract}

Keywords: adolescent; antiemetics; childhood cancer; chemotherapy-induced nausea and vomiting; olanzapine

\section{Introduction}

Chemotherapy-induced nausea and vomiting (CINV) is a frequent and potentially treatmentlimiting complication of cancer therapy in both adults and children [1]. Despite the appropriate use of prophylactic antiemetics, breakthrough and refractory CINV often occurs, for which there is no effective treatment in children $[2,3]$.

Olanzapine is well-known as an antipsychotic drug used to treat schizophrenia and depression in children and adolescents [4-7]. It has long been used in children because of its safety [8]. Olanzapine exerts antiemetic effects by acting on various neurotransmitters that cause vomiting and nausea [4]. Recent studies have investigated the use of olanzapine as an antiemetic drug (AED) in adult patients undergoing chemotherapy. The findings revealed an effective antiemetic effect compared with aprepitant or metoclopramide and other combinations of AEDs [9-15].

However, few studies have focused on olanzapine use in children $[2,3,16]$. In Korea, the Korea Food and Drug Administration permits olanzapine administration only for pediatric psychiatric diseases; therefore, there is no reported evidence of its use as an AED in pediatric patients with cancer. 
The purpose of this study was to investigate the efficacy and safety of off-label use of olanzapine as an AED in children and young adults with cancer being administered moderate and high emetogenic chemotherapy.

\section{Materials and Methods}

\subsection{Patients}

Data of children who were administered moderate and high emetogenic chemotherapy and olanzapine for CIV control at Yeungnam University Hospital from January 2018 to March 2020 were retrospectively analyzed. We reviewed the patients' medical, nursing, medication, and chemotherapy records, laboratory results, and vital charts. To investigate the adverse effects of olanzapine, we reviewed the nursing records for symptoms of pain, fatigue, somnolence, disturbed sleep, lack of appetite, dry mouth, or mood changes.

\subsection{Chemotherapy Emetogenicity Assessment}

The emetogenicity of chemotherapy drugs was classified according to the 2019 guideline for the classification of the acute emetogenic potential of antineoplastic medication in pediatric patients with cancer [17]. The emetogenic potential of anticancer drugs that are not described in this guideline was determined according to the 2011 guidelines [18].

\subsection{Standard CIV Prophylaxis}

Antiemetics practice was performed according to the POGO guidelines [19].

For blocks administered high emetogenic chemotherapy (HEC), dexamethasone (if corticosteroids were permitted), aprepitant (if $\geq 12$ years old) (Emend, MSD, Whitehouse Station, NJ, USA), and/or 5-HT3 antagonists (palonosetron; Aloxi, Helsinn Birex Pharmaceuticals Ltd., Dublin, Ireland) were used for CIV prophylaxis.

For blocks administered moderate emetogenic chemotherapy (MEC), dexamethasone (if corticosteroids were permitted) and/or 5-HT3 antagonists (palonosetron; Aloxi, Helsinn Birex Pharmaceuticals Ltd.Dublin, Ireland) were used for CIV prophylaxis.

\subsection{Olanzapine Treatment}

Olanzapine was administered for two different purposes: prophylaxis and rescue. First, it was administered for rescue; for patients in whom olanzapine was effective in rescue therapy, olanzapine prophylaxis was added from the next day or next chemotherapy block. For prophylaxis, olanzapine was orally administered $1 \mathrm{~h}$ prior to chemotherapy with or without other AEDs. In the rescue group, dexamethasone, 5-HT3 receptor antagonists, and aprepitant were used as prophylactic AEDs, but additional olanzapine was ultimately required for uncontrolled vomiting. When vomiting was not controlled by olanzapine, additional AEDs, such as metoclopramide, palonosetron, ondansetron, or diazepam were used. Olanzapine was initially administered orally at $0.1-0.14 \mathrm{mg} / \mathrm{kg} / \mathrm{dose}$ (maximum $10 \mathrm{mg} /$ dose) once daily. If there were no side effects, but nausea and vomiting occurred at night, olanzapine was administered twice daily. Dose modification was performed in $1.25 \mathrm{mg}$ increments while monitoring for antiemetic and adverse effects. For olanzapine, Zyprexa ( $2.5 \mathrm{mg}, 5 \mathrm{mg}$, and $10 \mathrm{mg}$; Lilly, Indianapolis, IN, USA) and Zyprexa zydis (5 mg and $10 \mathrm{mg}$; Lilly, Indianapolis, IN, USA) were used. Olanzapine was used as an antiemetic following approval for off-label drug use from the Health Insurance Review and Assessment Service and after receiving institutional review board approval.

\subsection{Response Assessment}

The CIV response of olanzapine was retrospectively assessed by reviewing the nursing and medication records. We counted the number of vomiting episodes in the nursing records and checked the number of additional AEDs used throughout the acute CIV phase (within $24 \mathrm{~h}$ after chemotherapy) 
in the medication records. We defined a complete response (CR) as no vomiting or additional AEDs recorded in the medical records throughout the acute phase. A partial response (PR) was defined as either vomiting more than once without additional AED use or additional AED use without vomiting. No response (NR) was defined as additional AED use and $>1$ episode of vomiting. Adverse events were graded according to National Cancer Institute Common Toxicity Criteria (version 4) [20].

\subsection{Ethical Approval}

This study was approved by the institutional review board of Yeungnam University Hospital (IRB No. YUMC 2019-08-014). All procedures performed in studies involving human participants were conducted in accordance with the ethical standards of the institutional research committee (Yeungnam University Hospital; IRB No. YUMC 2020-09-017).

\section{Results}

\subsection{Patient Characteristics}

Thirteen patients were included in this study (12 men, 1 woman). The average patient age at diagnosis was $13.2 \pm 4.8$ years. The patients had received diagnoses of Ewing's sarcoma, osteosarcoma, non-Hodgkin lymphoma, acute lymphocytic leukemia, Langerhans cell histiocytosis, neuroblastoma, acute myeloid leukemia, germ cell tumor, and synovial sarcoma (Table 1). Forty-one chemotherapy blocks were reviewed, of which $21(51.2 \%)$ blocks were high emetogenic cases and $20(48.8 \%)$ were moderate emetogenic cases (Table 1 ).

Table 1. Baseline characteristics of patients.

\begin{tabular}{ccc}
\hline & Characteristics & N (\%) \\
\hline \multirow{2}{*}{ Age } & Mean \pm SD & $13.2 \pm 4.8$ \\
& Median (range) & $15.1(4.0-18.0)$ \\
\hline \multirow{2}{*}{ Sex } & Male & $12(92.3)$ \\
& Female & $1(7.7)$ \\
\hline \multirow{4}{*}{ Diagnosis } & Ewing's sarcoma & $3(23.1)$ \\
& Acute lymphoblastic leukemia & $2(15.4)$ \\
& Acute myeloid leukemia & $2(15.4)$ \\
& Langerhans cell histiocytosis & $1(7.7)$ \\
& Neuroblastoma & $1(7.7)$ \\
& Synovial sarcoma & $1(7.7)$ \\
& Osteosarcoma & $1(7.7)$ \\
& Non-Hodgkin lymphoma & $1(7.7)$ \\
\hline
\end{tabular}

\subsection{Olanzapine Usage}

Twenty blocks (48.8\%) used olanzapine for prophylaxis and $21(51.2 \%)$ used olanzapine for rescue (Table 2). The duration of olanzapine administration was $3 \pm 2$ days at a dose of $0.07 \pm 0.04 \mathrm{mg} / \mathrm{kg} / \mathrm{dose}$ and $2.50 \pm 1.37 \mathrm{mg} / \mathrm{m}^{2} /$ dose. The median number of chemotherapy blocks that used olanzapine per patient was three (range, $1-16)$. Thirty-four (82.9\%) blocks used once-daily dosing and seven $(17.1 \%)$ used twice-daily dosing. 
Table 2. Olanzapine use during chemotherapy blocks.

\begin{tabular}{|c|c|c|}
\hline \multicolumn{2}{|c|}{ Characteristic } & \multirow{2}{*}{$\begin{array}{c}N(\mathbf{\%}) \\
21(51.2)\end{array}$} \\
\hline Chemotherany ametorenicity & High & \\
\hline cnemotnerapy emetogencity & Moderate & $20(48.8)$ \\
\hline \multirow{2}{*}{ Reason for olanzapine use } & Prophylaxis & $20(48.8)$ \\
\hline & Rescue & $21(51.2)$ \\
\hline \multirow{2}{*}{\multicolumn{2}{|c|}{$\begin{array}{l}\text { Chemotherapy block (days) } \\
\text { Duration of olanzapine use (days) }\end{array}$}} & $4 \pm 2$ \\
\hline & & $3 \pm 2$ \\
\hline \multirow{2}{*}{ Olanzapine dose } & $\mathrm{mg} / \mathrm{kg} / \mathrm{dose}$ & $0.07 \pm 0.04$ \\
\hline & $\mathrm{mg} / \mathrm{m}^{2} /$ dose & $2.50 \pm 1.37$ \\
\hline \multirow{2}{*}{ Olanzapine frequency } & Once daily & $34(82.9)$ \\
\hline & Twice daily & $7(17.1)$ \\
\hline
\end{tabular}

\subsection{Efficacy of Olanzapine}

In terms of overall CIV control, 31 (75.6\%) blocks achieved a CR, 6 (14.6\%) blocks achieved a PR, and 4 (9.8\%) blocks showed NR. In 35 of 41 blocks (87.4\%), no vomiting was recorded after olanzapine use. Two (4.9\%) patients vomited once, and four (9.6\%) patients vomited more than once after olanzapine administration.

CR was achieved after olanzapine administration in 19 of 21 blocks $(90.0 \%)$ of HEC; PR and NR were achieved for one block (4.8\%) each. Of the 20 blocks of MEC, CR was achieved in 12 blocks (60\%), PR in 5 blocks (25.0\%), and NR in 3 blocks (15.0\%) (Figure 1A).

(A)

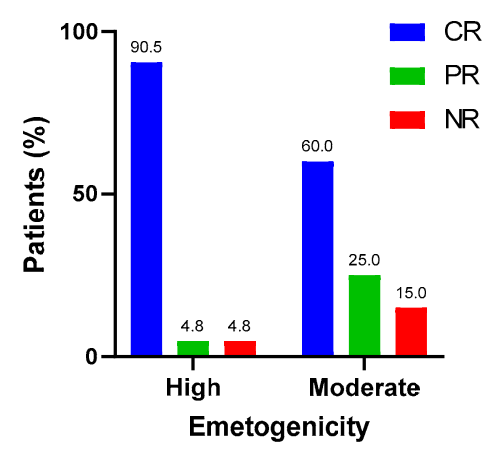

(C)

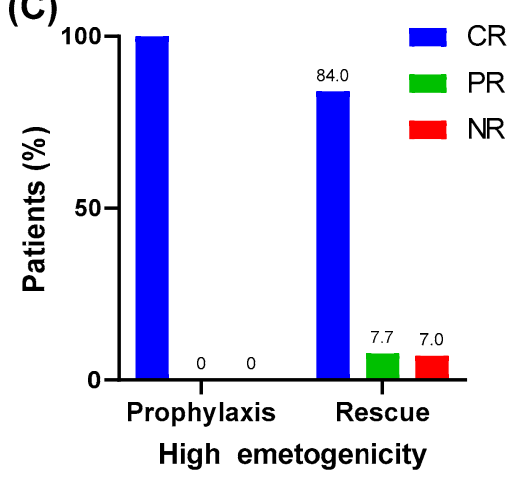

(B)

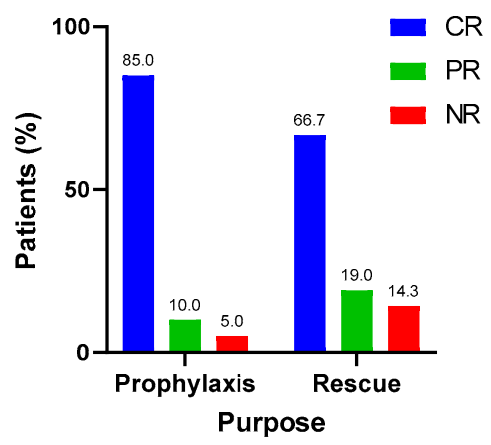

(D)

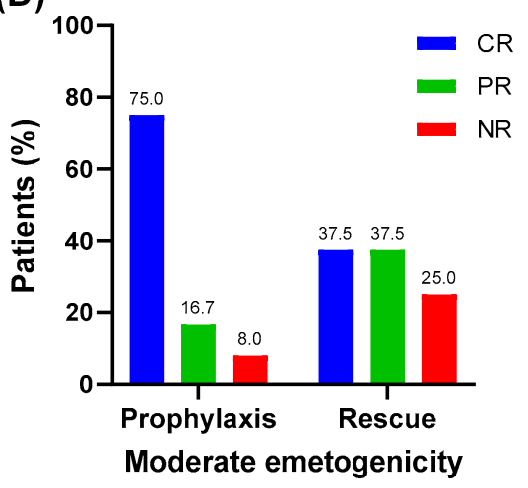

Figure 1. (A) Efficacy of olanzapine according to emetogenicity; (B) Efficacy of olanzapine according to purpose; (C) Efficacy of olanzapine according to purpose for high emetogenicity chemotherapy; (D) Efficacy of olanzapine according to purpose for moderate emetogenicity chemotherapy. CR, complete response; PR, partial response; NR, no response. 
CR was achieved in 17 of 20 blocks (85\%) when olanzapine was administered for prophylactic purposes; PR and NR were achieved in two (10\%) and one (5\%) blocks, respectively. When administered for rescue use, CR was achieved in 14 blocks (66.7\%), PR in 4 blocks (19.0\%), and NR in 3 (14.3\%) blocks (Figure 1B).

In HEC, all eight blocks showed a CR when olanzapine was used for prophylaxis. When olanzapine was administered as a rescue in HEC, $11(84.4 \%)$ blocks showed CR, 1 block (7.7\%) showed PR, and 1 block (7.7\%) showed NR (Figure 1C). In MEC, nine (75\%) blocks showed CR, two (16.7\%) blocks showed PR, and one block (8.3\%) showed NR when olanzapine was used as a prophylaxis. When olanzapine was used for a rescue purpose in MEC, three blocks (37.5\%) showed CR, two blocks (37.5\%) showed PR, and one block (25.0\%) showed NR (Figure 1D).

If there was a side effect of sedation due to taking it once a day, it was administered in two divided doses. In addition, one dose was effective, but if nausea and vomiting occurred again in the evening, it was administered once more. The resulting effect was good, and sedation side effects were reduced.

\subsection{Adverse Events during Olanzapine Treatment}

The most common adverse effect of patients was somnolence, and symptoms occurred in eight (19.5\%) blocks. Hyperglycemia occurred in six blocks (14.6\%) and fatigue and disturbed sleep occurred in one block $(2.4 \%)$ each.

\section{Discussion}

Despite the appropriate use of prophylactic antiemetics, breakthrough and refractory CIV often occurs, and there is a lack of information on its effectiveness in children. We determined the efficacy and safety of olanzapine for CIV control in 13 children undergoing 41 chemotherapy blocks at a single institution. When olanzapine was administered along with standard AEDs for CIV prophylaxis and rescue, $85 \%$ and $66.7 \%$ of children achieved complete CIV control, respectively. In addition, associated adverse events reported with its use were transient and clinically insignificant.

Flank et al. conducted a retrospective analysis of children $<18$ years old who were administered olanzapine for acute CINV control. In their study, the CR rate during HEC prophylaxis was $68 \%$. The olanzapine dose was only associated with a sedative effect but not with CIV control. The most reported adverse events were sedation (7\%) and increased transaminase levels (20\%) [3]. The mean initial single olanzapine dose administered was $0.10 \pm 0.051 \mathrm{mg} / \mathrm{kg} / \mathrm{dose}$, and the maximum single dose was $10 \mathrm{mg}$. In our study, CR rates of HEC prophylaxis and rescue were $100 \%$ and $84.0 \%$, respectively, and the most frequent adverse event was somnolence (15.6\%). The mean olanzapine dose was $0.07 \pm 0.04 \mathrm{mg} / \mathrm{kg} /$ dose $\left(2.50 \pm 1.37 \mathrm{mg} / \mathrm{m}^{2} /\right.$ dose $)$.

Flank et al. analyzed the initial dose of olanzapine in their study; in contrast, we analyzed the final dose, as dose modification was required to address sedation and unsatisfactory outcomes. The probable reason that the olanzapine dose was less than that reported by Flank et al. is that the dose was reduced owing to somnolence. Despite the low olanzapine dose, there may have been a racial difference in drug susceptibility, lessening the effect of antiemetics.

Venkatraman et al. conducted randomized phase III trial comparing olanzapine and metoclopramide for breakthrough CIV. CR rates were significantly higher in the olanzapine arm compared with the metoclopramide arm for nausea and vomiting. Hyperglycemia and drowsiness were more commonly seen in the olanzapine arm [16].

Some prospective trials have focused on olanzapine for CINV control in adult patients with cancer. Navari et al. conducted a randomized phase III trial to compare olanzapine and metoclopramide for breakthrough CINV in adult patients undergoing HEC. They found that olanzapine was better at controlling breakthrough emesis and nausea than metoclopramide [12]. In 2011, Navari et al. compared the effectiveness of olanzapine and aprepitant in adult patients undergoing HEC. Patients were randomly assigned to be administered olanzapine, palonosetron, dexamethasone (OPD) or aprepitant, palonosetron, and dexamethasone regimens. The CR rate was similar in both groups, 
but nausea control was better with the OPD regimen. Furthermore, the OPD regimen was more effective than the aprepitant, palonosetron, and dexamethasone regimen in controlling acute and delayed CINV [21].

Jeon et al. conducted a randomized, double-blind, placebo-controlled study focusing on whether olanzapine can reduce CINV in adult patients receiving MEC in Korea. Although they observed similar $\mathrm{CR}$ rates in the olanzapine and placebo groups, nausea control was better in the olanzapine group. In addition, they found that an OPD regimen significantly improved the quality of life of patients and reduced the frequency of vomiting [9].

In contrast to adults, there have been few prospective trials to evaluate olanzapine for CINV prevention in children. Flank et al. evaluated the feasibility of a trial assessing how olanzapine may contribute to CINV control in pediatric oncology patients. They enrolled 15 children who were administered at least half of the planned olanzapine dose $(0.14 \mathrm{mg} / \mathrm{kg} / \mathrm{dose}$; maximum $10 \mathrm{mg} / \mathrm{dose})$. Vomiting was completely controlled in eight patients in both the acute and delayed phases but 14 patients experienced nausea. They concluded that a pediatric trial of olanzapine for CINV control is feasible [2].

The limitation of this study is that the first is that it is a retrospective study involving a small number of patients, and this is considered a major limitation in drawing a clear conclusion. Second, the group of patients included in the study were patients with heterogenous characteristics, and the diagnosis was varied. Considering the differences between disease and its treatment, this is also a limitation of this study.

In this study, we retrospectively analyzed pediatric patients who were administered olanzapine for antiemetic purposes over a 26-month period at a single center. Olanzapine showed excellent efficacy in controlling CIV in pediatric patients who received MEC and HEC regimens. As this was a retrospective study, the analysis of nausea was insufficient. Nevertheless, this is the first study to demonstrate the efficacy of olanzapine for CINV control in Asian children. Our findings will contribute to the approval of olanzapine by the Korea Food and Drug Administration and national health insurance coverage for the treatment of and prophylaxis against CINV in pediatric patients with cancer in Korea and other countries.

\section{Conclusions}

Olanzapine, in combination with standard AEDs, showed promising results for both prophylaxis and rescue therapy of CINV in children. Furthermore, it showed few adverse effects, other than sedation, and was safe in children, which has long been recognized by psychiatrists. Olanzapine may be an important option for effectively controlling CINV in pediatric patients with cancer and subsequently improving their quality of life. There are limitations in reaching conclusions with small retrospective studies. Therefore, a prospective multicenter controlled study is needed to confirm these findings.

Author Contributions: J.M.L. contributed to the conception and design of this study; S.R.L., S.M.K. and M.Y.O. collected and analyzed data; S.R.L., S.M.K., and M.Y.O. wrote the manuscript; J.M.L. supervised the whole study process. All authors read and approved the final manuscript.

Funding: This research was funded by a 2019 Yeungnam University Research Grant.

Conflicts of Interest: The authors declare no conflict of interest.

\section{References}

1. Kang, H.J.; Loftus, S.; Taylor, A.; DiCristina, C.; Green, S.; Zwaan, C.M. Aprepitant for the prevention of chemotherapy-induced nausea and vomiting in children: A randomised, double-blind, phase 3 trial. Lancet Oncol. 2015, 16, 385-394. [CrossRef]

2. Flank, J.; Schechter, T.; Gibson, P.; Johnston, D.L.; Orsey, A.D.; Portwine, C.; Sung, L.; Dupuis, L.L. Olanzapine for prevention of chemotherapy-induced nausea and vomiting in children and adolescents: A multi-center, feasibility study. Support. Care Cancer 2018, 26, 549-555. [CrossRef] 
3. Flank, J.; Thackray, J.; Nielson, D.; August, A.; Schechter, T.; Alexander, S.; Sung, L.; Dupuis, L.L. Olanzapine for treatment and prevention of acute chemotherapy-induced vomiting in children: A retrospective, multi-center review. Pediatr. Blood Cancer 2015, 62, 496-501. [CrossRef]

4. Tanaka, K.; Inui, N.; Karayama, M.; Yasui, H.; Hozumi, H.; Suzuki, Y.; Furuhashi, K.; Fujisawa, T.; Enomoto, N.; Nakamura, Y.; et al. Olanzapine-containing antiemetic therapy for the prevention of carboplatin-induced nausea and vomiting. Cancer Chemother. Pharmacol. 2019, 84, 147-153. [CrossRef]

5. Krause, M.; Zhu, Y.; Huhn, M.; Schneider-Thoma, J.; Bighelli, I.; Chaimani, A.; Leucht, S. Efficacy, acceptability, and tolerability of antipsychotics in children and adolescents with schizophrenia: A network meta-analysis. Eur. Neuropsychopharmacol. 2018, 28, 659-674. [CrossRef]

6. Pagsberg, A.K.; Tarp, S.; Glintborg, D.; Stenstrom, A.D.; Fink-Jensen, A.; Correll, C.U.; Christensen, R. Acute Antipsychotic Treatment of Children and Adolescents With Schizophrenia-Spectrum Disorders: A Systematic Review and Network Meta-Analysis. J. Am. Acad. Child Adolesc. Psychiatry 2017, 56, 191-202. [CrossRef]

7. Strawn, J.R.; Delbello, M.P. Olanzapine for the treatment of bipolar disorder in children and adolescents. Expert Opin. Pharmacother. 2008, 9, 467-474. [CrossRef]

8. Flank, J.; Sung, L.; Dvorak, C.C.; Spettigue, W.; Dupuis, L.L. The safety of olanzapine in young children: A systematic review and meta-analysis. Drug Saf. 2014, 37, 791-804. [CrossRef]

9. Jeon, S.Y.; Han, H.S.; Bae, W.K.; Park, M.R.; Shim, H.; Lee, S.C.; Go, S.I.; Yun, H.J.; Im, Y.J.; Song, E.K. A Randomized, Double-Blind, Placebo-Controlled Study of the Safety and Efficacy of Olanzapine for the Prevention of Chemotherapy-Induced Nausea and Vomiting in Patients Receiving Moderately Emetogenic Chemotherapy: Results of the Korean South West Oncology Group (KSWOG) Study. Cancer Res. Treat. 2019, 51, 90-97. [CrossRef]

10. Dulal, S.; Paudel, B.D.; Neupane, P.; Shah, A.; Acharya, B.; Poudyal, B.S.; Shilpakar, R.; Wood, L.A. Randomized Phase II Trial to Compare the Efficacy of Haloperidol and Olanzapine in the Control of Chemotherapy-Induced Nausea and Vomiting in Nepal. J. Glob. Oncol. 2019, 5, 1-6. [CrossRef]

11. Mehra, N.; Ganesan, P.; Ganesan, T.S.; Veeriah, S.; Boopathy, A.; Radhakrishnan, V.; Dhanushkodi, M.; Rajaraman, S.; Ganesharajah, S.; Sagar, T.G. Effectiveness of olanzapine in patients who fail therapy with aprepitant while receiving highly emetogenic chemotherapy. Med. Oncol. 2017, 35, 12. [CrossRef]

12. Navari, R.M.; Nagy, C.K.; Gray, S.E. The use of olanzapine versus metoclopramide for the treatment of breakthrough chemotherapy-induced nausea and vomiting in patients receiving highly emetogenic chemotherapy. Support. Care Cancer 2013, 21, 1655-1663. [CrossRef]

13. Navari, R.M.; Einhorn, L.H.; Loehrer, P.J., Sr.; Passik, S.D.; Vinson, J.; McClean, J.; Chowhan, N.; Hanna, N.H.; Johnson, C.S. A phase II trial of olanzapine, dexamethasone, and palonosetron for the prevention of chemotherapy-induced nausea and vomiting: A Hoosier oncology group study. Support. Care Cancer 2007, 15, 1285. [CrossRef]

14. Navari, R.M.; Einhorn, L.H.; Passik, S.D.; Loehrer, P.J., Sr.; Johnson, C.; Mayer, M.L.; McClean, J.; Vinson, J.; Pletcher, W. A phase II trial of olanzapine for the prevention of chemotherapy-induced nausea and vomiting: A Hoosier Oncology Group study. Support. Care Cancer 2005, 13, 529-534. [CrossRef]

15. Navari, R.M.; Qin, R.; Ruddy, K.J.; Liu, H.; Powell, S.F.; Bajaj, M.; Dietrich, L.; Biggs, D.; Lafky, J.M.; Loprinzi, C.L. Olanzapine for the Prevention of Chemotherapy-Induced Nausea and Vomiting. N. Engl. J. Med. 2016, 375, 134-142. [CrossRef]

16. Radhakrishnan, V.; Pai, V.; Rajaraman, S.; Mehra, N.; Ganesan, T.; Dhanushkodi, M.; Perumal Kalaiyarasi, J.; Rajan, A.K.; Selvarajan, G.; Ranganathan, R.; et al. Olanzapine versus metoclopramide for the treatment of breakthrough chemotherapy-induced vomiting in children: An open-label, randomized phase 3 trial. Pediatr. Blood Cancer 2020, 67, e28532. [CrossRef]

17. Paw Cho Sing, E.; Robinson, P.D.; Flank, J.; Holdsworth, M.; Thackray, J.; Freedman, J.; Gibson, P.; Orsey, A.D.; Patel, P.; Phillips, R.; et al. Classification of the acute emetogenicity of chemotherapy in pediatric patients: A clinical practice guideline. Pediatr. Blood Cancer 2019, 66, e27646. [CrossRef]

18. Dupuis, L.L.; Boodhan, S.; Sung, L.; Portwine, C.; Hain, R.; McCarthy, P.; Holdsworth, M.; Pediatric Oncology Group of Ontario. Guideline for the classification of the acute emetogenic potential of antineoplastic medication in pediatric cancer patients. Pediatr. Blood Cancer 2011, 57, 191-198. [CrossRef] 
19. Dupuis, L.L.; Boodhan, S.; Holdsworth, M.; Robinson, P.D.; Hain, R.; Portwine, C.; O'Shaughnessy, E.; Sung, L.; Pediatric Oncology Group of Ontario. Guideline for the prevention of acute nausea and vomiting due to antineoplastic medication in pediatric cancer patients. Pediatr. Blood Cancer 2013, 60, 1073-1082. [CrossRef]

20. Zanglis, A.; Valsamaki, P.; Fountos, G. Erdheim-Chester disease: Symmetric uptake in the (99m)Tc-MDP bone scan. Hell. J. Nucl. Med. 2008, 11, 164-167.

21. Navari, R.M.; Gray, S.E.; Kerr, A.C. Olanzapine versus aprepitant for the prevention of chemotherapy-induced nausea and vomiting: A randomized phase III trial. J. Support Oncol. 2011, 9, 188-195. [CrossRef]

(C) 2020 by the authors. Licensee MDPI, Basel, Switzerland. This article is an open access article distributed under the terms and conditions of the Creative Commons Attribution (CC BY) license (http://creativecommons.org/licenses/by/4.0/). 